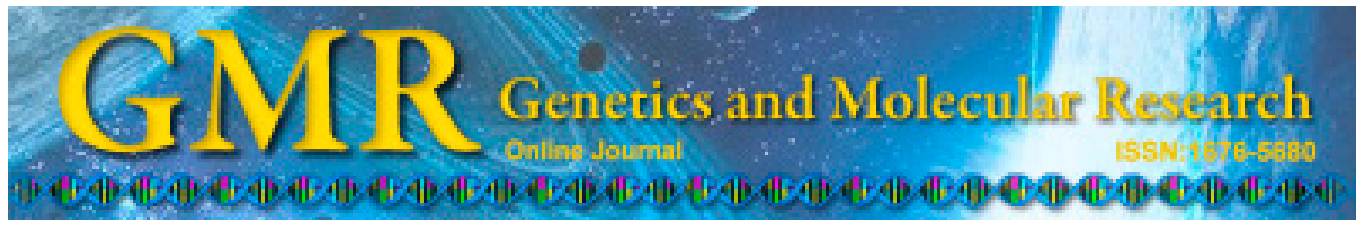

\title{
Relationship between DNA repair gene XPD751 single-nucleotide polymorphisms and prognosis of colorectal cancer
}

\author{
Y. Dong, J.W. Liu, Y.J. Gao, T. Zhou and Y.M. Chen \\ First Affiliated Hospital of Medical University, Dalian, China \\ Corresponding author: Y.M. Chen \\ E-mail: dlchenyamin@126.com / dongyang979828@hotmail.com
}

Genet. Mol. Res. 14 (2): 5390-5398 (2015)

Received May 19, 2014

Accepted October 28, 2014

Published May 22, 2015

DOI http://dx.doi.org/10.4238/2015.May.22.8

\begin{abstract}
We examined the relationships between singlenucleotide polymorphisms (SNPs) in the DNA repair gene XPD751 and the efficacy and time to disease progression (TTP) in colorectal cancer patients after platinum-based chemotherapy. Ninety-eight patients diagnosed with advanced colorectal cancer were subjected to oxaliplatin and 5-fluorouracil combination therapy. DNA was extracted from venous blood before chemotherapy. Polymerase chain reactionrestriction fragment length polymorphism analysis was used to detect XPD751 SNPs. The relationship between genotypes and prognosis was compared. The frequencies of the XPD751 Lys/Lys, Lys/Gln, and Gln/Gln genotypes were 76 (77.55\%), $17(17.35 \%)$, and $5(5.10 \%)$, respectively. The efficiency of XPD751 Lys/Lys, Lys/Gln and Gln/Gln genotypes were $50.00,29.41$, and $20 \%$, respectively. The efficiency rate between XPD751 Lys/Lys and Lys/Gln showed a significant difference $\left(\chi^{2}=4.04, \mathrm{P}<0.05\right)$. After adjusting for gender, age, and metastasis location, chemotherapy failure in patients carrying XPD751 Lys/Gln was 3.404-fold higher than in patients carrying the Lys/ Lys genotype. Median TTP was 304 days (10.1 months) and median TTP in patients with XPD751 Lys/Lys and $\geq 1$ Gln genotype was 340 and 87 days. After comparing TTP in patients carrying Lys/Lys and
\end{abstract}


patients carrying $\geq 1 \mathrm{Gln}$, the difference was significant. SNPs in the DNA repair gene XPD751 may be associated with oxaliplatin and 5 -fluorouracil chemotherapy sensitivity in colorectal cancer patients. These polymorphisms may be associated with TTP in patients with advanced colorectal cancer after first-line chemotherapy of oxaliplatin. XPD751 SNPs may be predictive factors of prognosis in colorectal cancer patients receiving oxaliplatin and 5-fluorouracil chemotherapy.

Key words: Colorectal cancer; Disease progression time; Drug efficacy; Repair genes; XPD751

\section{INTRODUCTION}

Colorectal cancer (CRC) is a common gastrointestinal cancer, and CRC mortality has increased in the urban and rural areas of China (Chen et al., 2006). With diet and lifestyle changes, CRC incidence and mortality is expected to continue to increase in China to become one of the most prevalent types of cancer (Zhen and Cai, 2004). Thus, it is very important to understand the pathogenesis and improve treatment of colorectal cancer. The xeroderma pigmentosum (XPD) gene is associated with nucleotide excision repair (NER) functional changes, which may affect the efficacy and prognosis of platinum chemotherapy. The incidence of CRC in our country is increasing at an average annual growth rate of $4.2 \%$, making it a serious threat to human health. Chemotherapy plays an important role in the comprehensive treatment of CRC. An oxaliplatin and FOLFOX regimen consisting of 5-fluorouracil (5-Fu) is currently the most preferred chemotherapy regimen for CRC treatment (Sun et al., 2007). Studies have shown that single-nucleotide polymorphisms in the human DNA repair gene XPD751 are associated with the sensitivity of platinum drugs (Gurubhagavatula et al., 2004). However, in different CRC patients receiving the same chemotherapy regimen, the efficacy and tolerability of treatment, disease progression time, and survival time show large differences. These differences cannot be explained with regards to age, concomitant diseases, concomitant medications, liver and kidney function status, and other factors. DNA damage repair, drug metabolism, and detoxification gene polymorphisms affect the sensitivity and toxicity of drugs in different individuals. Studies have shown that low levels of DNA damage repair in tumor tissue can reduce platinum drug and DNA adduct resection as well as enhance chemosensitivity, which may improve the prognosis of platinum-based chemotherapy (Quintela-Fandino et al., 2006). NER is the key process in DNA repair, and XPD751 plays an important role in this pathway by affecting the sensitivity of tumor cells to platinum drugs. Single-nucleotide polymorphisms (SNPs) can be used to predict prognosis in patients receiving platinum-based chemotherapy. SNPs have varying effects in different races. In this study, we examined the relationship between these polymorphisms and treatment efficacy and time to disease progression (TTP) in Chinese patients with advanced $\mathrm{CRC}$ receiving the platinum-based chemotherapy.

\section{MATERIAL AND METHODS}

\section{Subjects}

From 2005-2010, 98 patients with advanced CRC who received pathological diagno- 
sis and therapy in our hospital were collected. DNA was extracted from venous blood; 60 cases were male and 38 cases were female. They were aged from $42-74$ years with a median age of 57 years. Fifty-five cases had colon cancer and 43 cases had CRC. A total of 33 cases had liver metastasis, 20 cases had liver metastasis and abdominal lymph node metastasis, 12 cases had liver and lung metastases, 8 cases had lung metastasis, 18 cases had abdominal lymph node metastasis, and 7 cases had abdominal lymph node metastasis and ascites. All cases selected were confirmed by computed tomography or magnetic resonance imaging scanning to measure lesion diameter, which was greater than $10 \mathrm{~mm}$. Before chemotherapy, routine blood tests and liver and kidney functions were normal. There were no significant electrocardiogram abnormalities and Karnofskyp scores were all greater than 70 points.

\section{Chemotherapy}

All enrolled patients were given FOLFOX chemotherapy, which included $85 \mathrm{mg} / \mathrm{m}^{2}$ $\mathrm{d}_{1}$ oxaliplatin, $200 \mathrm{mg} / \mathrm{m}^{2} \mathrm{~d}_{1-2}$ leucovorin (CF), $400 \mathrm{mg} / \mathrm{m}^{2} \mathrm{~d}_{1-2} 5$-Fu for ivgtt, $600 \mathrm{mg} / \mathrm{m}^{2} \mathrm{~d}_{1-2}$ 5 -Fu for 22-h continuous infusion. Each treatment cycle was 2 weeks.

\section{Efficacy evaluation and outcome measurements}

DNA was extracted from 98 subjects who had completed 4-6 cycles of chemotherapy, and efficacy evaluation was performed. TTP was observed from the start of chemotherapy until the first confirmed disease progression or death; 16 patients did not show TTP until the end of the follow-up and these data were censored.

\section{Specimen collection and experimental methods}

Before chemotherapy, $2 \mathrm{~mL}$ peripheral venous blood was taken from all patients. After sodium citrate anticoagulation treatment, the samples were stored at $-20^{\circ} \mathrm{C}$. DNA was extracted from anticoagulated blood using a DNA extraction kit from Beijing Baitaike Biotechnology Co., Ltd. (Beijing, China). The polymerase chain reaction (PCR)-restriction fragment length polymorphism technique was used to amplify the DNA repair gene XPD751, containing Lys751Gln polymorphic gene fragment (436-bp), and the following primers for XPD751 were used for detection: 5'-GCCCGCTCTGGATTATACG-3' and 5'-CTATCATCTCCTGGC CCCC-3'. The $25-\mu \mathrm{L}$ PCR system contained $5 \mu \mathrm{L}$ template DNA, 10 pmol of each primer, $1 \mu \mathrm{L}$ dNTPs, $0.2 \mu \mathrm{L}$ ExTaq polymerase, $2.5 \mu \mathrm{L} 10 \mathrm{X}$ PCR buffer, $1.5 \mu \mathrm{L} \mathrm{MgCl}_{2}$. The PCR conditions were $94^{\circ} \mathrm{C}$ denaturing for $2 \mathrm{~min}, 94^{\circ} \mathrm{C}$ for $30 \mathrm{~s}, 62^{\circ} \mathrm{C}$ for $30 \mathrm{~s}$, and $72^{\circ} \mathrm{C}$ for $30 \mathrm{~s}$. After 35 cycles, amplified products were extended for $7 \mathrm{~min}$ at $72^{\circ} \mathrm{C}$. Next, $5 \mu \mathrm{L}$ PCR product was digested with the restriction enzyme $P s t$ at $37^{\circ} \mathrm{C}$ overnight, and $3 \%$ agarose gel electrophoresis analysis was performed to detect the XPD751 genotypes. All reagents and primers were purchased from Takara Bio (Shiga, Japan). DNA markers were purchased from Brad Company (Yeh et al., 2005). The results are shown in Figure 1.

Wild-type 751 Lys/Lys PCR products contained 1 Pst restriction site, generating 2 fragments of 290 and $146 \mathrm{bp}$. Two restriction sites were generated after Lys $\rightarrow$ Gln mutation, and the homozygous mutant $\mathrm{Gln} / \mathrm{Gln}$ produced 3 fragments of 227, 146, and $63 \mathrm{bp}$. The heterozygous mutant Lys/Gln produced 4 fragments of 90, 227, 146, and $63 \mathrm{bp}$. 


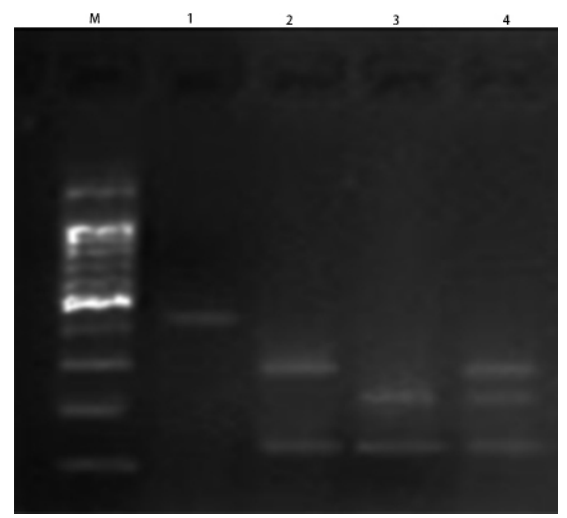

Figure 1. Lys751Gln PCR products of the XPD gene and electrophoresis patterns after Pst I digestion. (Note: lane $M=$ Marker; lane 1 = 436-bp fragment; lane 2 = Lys/Lys genotype (A/A); lane $3=\mathrm{Gln} / \mathrm{Gln}$ genotype $(\mathrm{C} / \mathrm{C})$; lane $4=\mathrm{Lys} / \mathrm{Gln}$ genotype $(\mathrm{A} / \mathrm{C})$.

\section{Statistical methods}

The SPSS 17.0 statistical software was used for statistical analysis (SPSS, Inc., Chicago, IL, USA), while the $\chi^{2}$ test was used to detect the chemotherapy genotypes differences in the DNA repair gene XPD751. The unconditional logistic regression model was used to calculate the odds ratio (OR) and $95 \%$ confidence intervals $(95 \% \mathrm{CI})$ to indicate chemosensitivity differences among different genotypes. All tests were 2 -sided, and $\mathrm{P}<0.05$ was considered to be statistically significant. MTTP was calculated using the Kaplan-Meier method. Log-rank test was used to compare MTTP among different genotypes. Cox proportional hazards model was used to assess the impact of various factors affecting the results of the experiment and the $95 \% \mathrm{CI}$ was calculated. All $\mathrm{P}$ values were 2 -tailed, and $\mathrm{P}<0.05$ was considered to be statistically significant.

\section{Evaluation criteria of chemotherapy efficacy}

The evaluation criteria were based on the recent RECIST objective efficacy evaluation of solid tumors, divided into complete remission (CR), partial remission (PR), stable (SD) and progression (PD), and the CR + PR were considered effective (RR). Evaluation included the following: 1) CR of target lesion: disappearance of all target lesions. PR: Sum of the baseline lesion diameter narrowing reached 30\%. PD: Total increase of baseline lesion diameter was more than $20 \%$ or new lesions. SD: Total lesion diameter baseline shrank but did not reach PR or increased but did not reach PD. 2) Evaluation of non-target lesion: CR: All non-target lesions disappeared and tumor marker levels were normal. SD: One or more non-target lesions and/or tumor markers persisted or were higher than normal levels. PD: One or more new lesions and/or non-target lesions progression.

\section{RESULTS}

\section{SNP distribution in DNA repair genes XPD751}

The genotype frequencies in the DNA repair gene XPD751 were: 76 with Lys/Lys 
(77.55\%), 17 with Lys/Gln, and 5 with $\mathrm{Gln} / \mathrm{Gln}(22.45 \%)$.

\section{Association between the DNA repair gene XPD751 SNPs and chemotherapy efficacy}

Complete remission was not observed in any of the 98 patients, 0 cases had CR, 44 cases had PR (44.90\%), 34 cases had SD (34.70\%), and 20 cases had PD (20.41\%). The effective rate of those carrying XPD751 Lys/Lys was $50.00 \%$, while the effective rate carrying Lys/Gln was $29.41 \%$ and the effective rate carrying Gln/Gln was $20.00 \%$. Patients were divided into groups according to treatment effectiveness ( $\mathrm{CR}+\mathrm{PR})$. The chemotherapy effectiveness rates for the XPD751 A/A, A/C, and C/C genotypes were 38 patients $(50 \%)$, 5 cases $(29.41 \%)$, and 1 case $(20.00 \%)$, respectively. The frequency of genotype Gln/Gln was low in China $(8,20,22)$, and the number of cases collected in this study was limited. Therefore, the expression of the Gln/Gln genotype was rare. This experiment only found that there were differences between the efficiency of Lys/Lys and Lys/Gln, and that these differences were significant $\left(\chi^{2}=4.04, \mathrm{P}<0.05\right)$, as shown in Table 1 . In logistic regression, after adjusting for gender, age, and other factors in this group of patients, the XPD751 genotype remained significantly associated with chemosensitivity $(\mathrm{P}<0.05)$, and the $\mathrm{OR}=$ 3.404 suggested that individual chemosensitivity in patients carrying the A/A genotype was 3.404 -fold higher than in patient carrying the $\mathrm{A} / \mathrm{C}$ genotype in the XPD751 codon $(95 \% \mathrm{CI}$ $=1.819-6.369$; Table 2 and 3 ).

Table 1. Association between XPD751 SNPs and efficacy of chemotherapy in 98 cases of advanced colorectal cancer $(\mathrm{N}=98$ patients).

\begin{tabular}{lccrrrr}
\hline Genotype & CR & PR & SD & PD & n & $\chi^{2}$ \\
\hline Lys/Lys & 0 & 38 & 26 & 12 & 76 & P value \\
Lys/Gln & 0 & 5 & 6 & 6 & 17 & 4.04 \\
Gln/Gln & 0 & 1 & 2 & 2 & 5 & $<0.05$ \\
\hline
\end{tabular}

Table 2. Characteristics of patients and comparison of efficacy $(\mathrm{N}=98)$.

\begin{tabular}{|c|c|c|c|c|c|c|}
\hline & $\mathrm{CR}+\mathrm{PR}$ & SD & PD & $\mathrm{N}$ & $\chi^{2}$ & $\mathrm{P}$ value \\
\hline \multicolumn{7}{|l|}{ Gender } \\
\hline Man & 27 & 21 & 12 & 60 & \multirow[t]{2}{*}{0.011} & \multirow[t]{2}{*}{0.917} \\
\hline Female & 17 & 13 & 8 & 38 & & \\
\hline \multicolumn{7}{|l|}{ Age (years) } \\
\hline$<57$ & 20 & 15 & 14 & 49 & \multirow[t]{2}{*}{4.257} & \multirow[t]{2}{*}{0.039} \\
\hline$>57$ & 24 & 20 & 5 & 49 & & \\
\hline \multicolumn{7}{|l|}{ Tumor metastasis } \\
\hline Liver metastases & 18 & 11 & 4 & 33 & \multirow[t]{6}{*}{9.397} & \multirow[t]{6}{*}{0.094} \\
\hline Liver metastasis with abdominal lymph node metastasis & 10 & 8 & 2 & 20 & & \\
\hline Liver metastasis with pulmonary metastasis & 5 & 4 & 3 & 12 & & \\
\hline Lung metastases & 4 & 3 & 1 & 8 & & \\
\hline Abdominal lymph node metastasis & 5 & 6 & 7 & 18 & & \\
\hline Abdominal lymph node metastasis with ascites & 2 & 2 & 3 & 7 & & \\
\hline \multicolumn{7}{|l|}{ Genotype } \\
\hline Lys/Lys & 38 & 26 & 12 & 76 & \multirow{3}{*}{5.831} & \multirow{3}{*}{0.016} \\
\hline Lys/Gln & 5 & 6 & 6 & 17 & & \\
\hline $\mathrm{G} \ln / \mathrm{Gln}$ & 1 & 2 & 2 & 5 & & \\
\hline
\end{tabular}


Table 3. Association between XPD751 polymorphisms and median TTP.

\begin{tabular}{|c|c|c|c|c|c|c|}
\hline \multirow[t]{2}{*}{ Genotype } & \multirow{2}{*}{$\begin{array}{c}\text { MTTP } \\
\text { (95\%CI, day) }\end{array}$} & \multicolumn{2}{|c|}{ Log-rank test } & \multicolumn{2}{|c|}{ Cox regression } & \multirow[t]{2}{*}{$P$} \\
\hline & & $\chi^{2}$ & $\mathrm{P}$ & OR & $95 \% \mathrm{CI}$ & \\
\hline Lys/Lys & $340(306.979-373.235)$ & 21.089 & $<0.05$ & 3.404 & $(1.819-6.369)$ & $<0.05$ \\
\hline Lys/Gln or Gln/Gln & $87(71.652-102.348)$ & & & & & \\
\hline Total & $304(280.610-327.390)$ & & & & & \\
\hline
\end{tabular}

\section{Prognosis status in different $X P D 751$ genotypes}

The median follow-up time for the 98 patients was 480 (90-1200) days. Post-treatment median TTP was 304 days (10.1 months). The median TTPs for different genotypes were compared; the XPD751 Lys/Lys gene frequency included 76 cases $(77.55 \%)$, with a median TTP of 340 days (11.3 months). The genotype frequency of Lys/Gln and Gln/Gln was 22 (22.45\%), with a median TTP of 87 days (2.9 months). The median TTP between Lys/Lys and patients with at least $1 \mathrm{Gln}$ was statistically significant $\left(\chi^{2}=21.089, \mathrm{P}<0.05\right)$, suggesting that oxaliplatin shows better prognosis in patients with the Lys/Lys genotype (Table 3 and Figure 2).

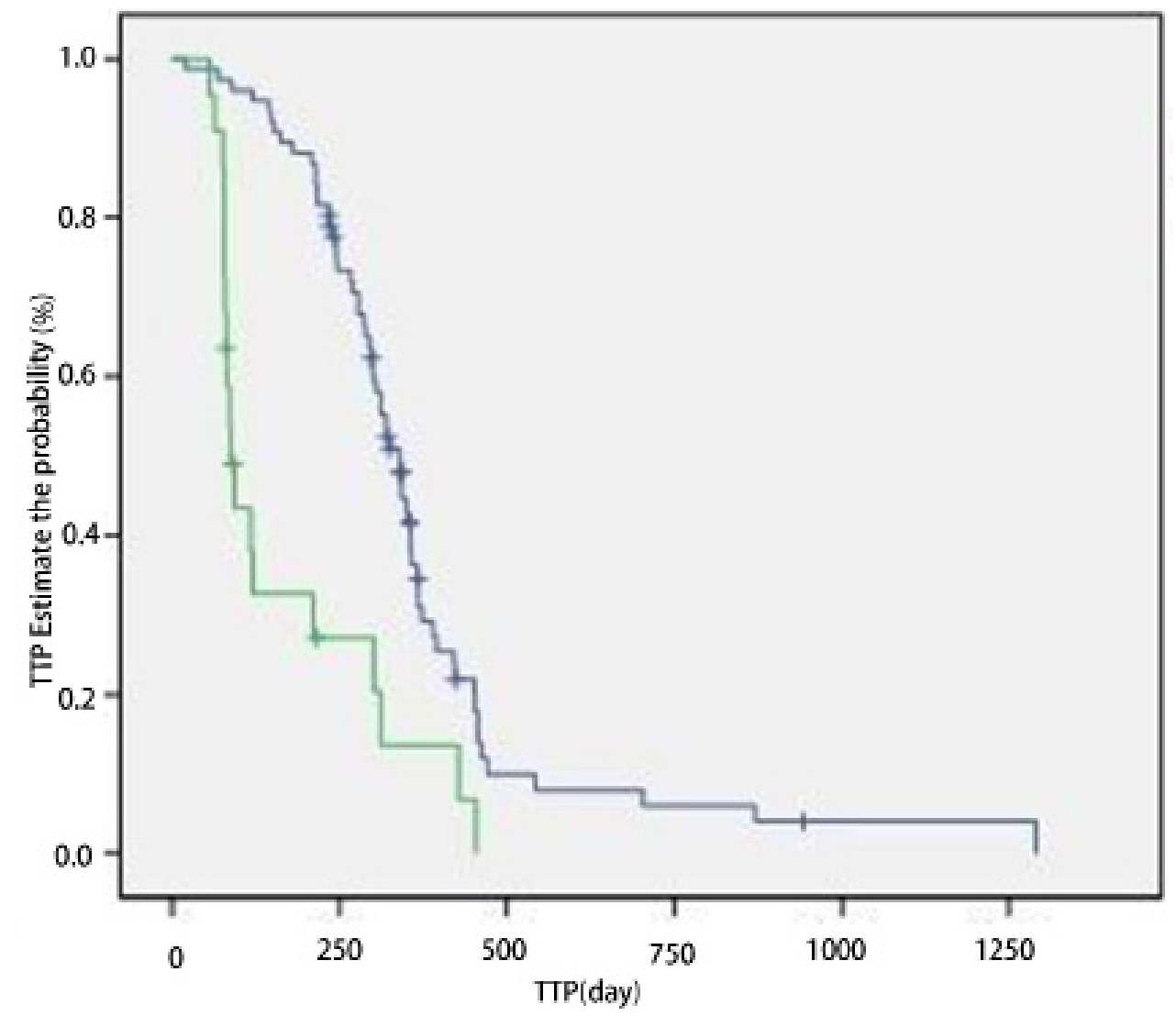

Figure 2. Association between XPD polymorphisms and median TTP. 


\section{DISCUSSION}

The NER system is an important component of the human DNA repair system, and platinum-induced DNA damage is typically repaired by this system. Cytotoxicity of platinum drugs to tumor cells occurs through binding the nucleophilic cell DNA to form platinum-DNA adducts, leading to crosslinking within the chain-stranded DNA and inducing DNA damage and cell death. This can affect the body's sensitivity to platinum-based chemotherapy. Thus, differences in DNA damage repair capacity may be an important factor determining platinum drug efficacy (Rosell et al., 2002; Guo et al., 2009). The XPD gene is an important member of the NER system and is located on the long arm of chromosome 19. It is mainly involved in NER and transcription, identification, and repair of a wide range of damage unrelated to gene structure; it can also remove a variety of types of DNA damage in the body. Amino acid sequence changes may affect the interactions between different proteins, leading to individual differences in repair capacity (Matullo et al., 2001). The XPD-encoded protein is ATP-dependent evolutionary conserved DNA helicase, an important component of the type II transcription factor $\mathrm{H}$ composite (TFIIH), which participates in NER and gene transcription. The mutation frequency of codons 312 and 751 in the XPD gene were high. Asp312Asn $(\mathrm{G} \rightarrow \mathrm{A})$ and Lys751Gln $(\mathrm{A} \rightarrow \mathrm{C})$ were intentional mutation causing amino acid changes and affecting the function of DNA repair pathways. Asp312Asn is highly conserved in evolution and may play a role in maintaining XPD protein function. Lys751Gln is located at the N-terminus of the XPD protein and is not well-conserved, and thus can be used as an indicator of DNA repair capacity.

A previous study confirmed that the codon $751 \mathrm{C} \rightarrow \mathrm{A}$ polymorphism in XPD may cause the amino acid substitution Lys $751 \rightarrow$ Gln 751 , which was closely associated with individual repair differences. This can increase the incidence and risk of cancer and affect tumor sensitivity to anti-cancer drugs as well as the prognosis of patients (Zárate et al., 2006). A study by Paré et al. (2008) found that XPD751 SNPs affected the prognosis of patients with advanced CRC receiving oxaliplatin treatment. The progression-free survival time in patients with the Lys/Lys genotype was 12 months, while the median progression-free survival was 8 months in patients with the Lys/Gln and Gyn/Gln genotypes. For median overall survival, the difference was also significant, and the median overall survival in patients with the A/A genotype was 41 months; the median overall survival for patients with the other 2 genotypes was 17 months, and this difference was statistically significant. Le Morvan et al. (2007) observed similar results; event-free survival differed in for different XPD751 genotypes, and the median event-free survival and overall survival in patients carrying at least 1 mutant allele was shorter than that in patients carrying the wild-type genotype. Park et al. (2001) found that in 73 CRC patients with metastatic joint treatment with 5-Fu and oxaliplatin, patients carrying the XPD751 Lys/Lys genotype had a median survival of 17.4 months, while the median survival time of patients with the Lys/Gln and Gln/Gln genotypes was 12.8 and 3.3 months, respectively; this difference was significant. The prognosis of patients with SNPs in the XPD312 gene showed no difference. Most previous studies are consistent with our results, and the survival time in patients carrying the wild-type allele of XPD751 was longer than that in patients carrying a mutation of any allele. The present study showed that patients carrying the XPD751 Lys/Lys genotype were more likely to have an increased risk of lung cancer and were more sensitive to chemotherapy. Chen et al. (2002) found that the XPD751 Lys gene was positively correlated with lung cancer risk. Lunn et al. (2000) reported that the XPD751 Lys/Lys genotype can increase the level of chromosomal aberrations and reduce the ability to repair DNA 
damage. The Spanish Lung Cancer Collaborative Group found that in 52 patients with phase IV lung cancer receiving platinum-based chemotherapy, the median TTP for those with the XPD751 Lys/Lys genotype was longer, and survival was poor in patients carrying the Gln/Gln genotype (Viñolas et al., 2003). It has been hypothesized that patients carrying the XPD751 Gln allele had low DNA repair capacity (Hemminki et al., 2001; Spitz et al., 2001; Au et al., 2003). Yin et al. (2006) showed that lung cancer risk in non-smoking women carrying the XPD751 Lys/Gln or Gln/Gln genotype was 2.80 -fold higher than that in non-smoking women carrying the Lys/Lys genotype. Sarries et al. (2003) reported that patients carrying the XPD751 Lys/Gln genotype had the best prognosis when receiving gemcitabine/cisplatin chemotherapy, the prognosis for the Lys/Lys was acceptable, while the prognosis for Gln/Gln was the worst.

We found that in 98 advanced CRC patients receiving FOLFOX chemotherapy, the objective response rates in patients carrying the XPD751 Lys/Lys and Lys/Gln genotypes were 50.00 and $29.41 \%$, respectively. The possibility of chemotherapy failure in patients carrying the XPD751 Lys/Gln genotype was 3.404-fold higher than that in patients carrying the Lys/ Lys genotype. The median TTP in patients carrying the Lys/Lys genotype was 340 days (11.3 months), which was significantly higher than that in patients carrying the XPD751 Lys/Gln or Gln/Gln genotype, with a median TTP of 87 days (2.9 months) $\left(\chi^{2}=21.089, \mathrm{P}<0.05\right)$. Patients carrying the XPD751 Lys/Lys genotype were more sensitive to platinum-based chemotherapy, and the median TTP was longer, which is consistent with the results of a previous study (Lou et al., 2006). SNPs in DNA repair genes affect the sensitivity to the drugs in different individuals. Gender, age, and metastasis sites were not significantly associated with prognosis, but SNPs in the DNA repair gene XPD751 were associated with chemotherapy prognosis of CRC patients receiving FOLFOX. The prognosis for patients with the wild-type genotype was better. The results of the present study were not completely consistent, which may be due to ethnicity, lifestyle, environmental factors, and various family hereditary diseases; tumor sensitivity to chemotherapy may be affected by the interaction of multi factors. Therefore, multi-gene studies are more suitable for evaluating the selection and prognosis of chemotherapy.

With the standardization of detection technology and testing, detecting SNPs in the peripheral blood DNA repair gene XPD751 may be useful for predicting the sensitivity to chemotherapeutic drugs and disease progression time in different CRC patients. These methods may also provide a basis for individualized treatment of CRC patients.

\section{REFERENCES}

Au WW, Salama SA and Sierra-Torres CH (2003). Functional characterization of polymorphisms in DNA repair genes using cytogenetic challenge assays. Environ. Health Perspect. 111: 1843-1850.

Chen K, Song L, Jin MJ, Fan CH, et al. (2006). Relationship folate metabolic enzyme gene polymorphisms and colorectal cancer susceptibility. Chin. J. Oncol. 28: 429-432.

Chen S, Tang D, Xue K, Xu L, et al. (2002). DNA repair gene XRCC1 and XPD polymorphisms and risk of lung cancer in a Chinese population. Carcinogenesis 23: 1321-1325.

Guo J, Liu L and Wang ZH (2009). XPD genetic polymorphisms for late-stage colorectal carcinoma with the research about the relation between the platinum drugs sensitivity. Chin. J. Gerontol. 29: 595-596.

Gurubhagavatula S, Liu G, Park S, Zhou W, et al. (2004). XPD and XRCC1 genetic polymorphisms are prognostic factors in advanced non-small-cell lung cancer patients treated with platinum chemotherapy. J. Clin. Oncol. 22: 2594-2601.

Hemminki K, Xu G, Angelini S, Snellman E, et al. (2001). XPD exon 10 and 23 polymorphisms and DNA repair in human skin in situ. Carcinogenesis 22: 1185-1188.

Le Morvan V, Smith D, Laurand A, Brouste V, et al. (2007). Determination of ERCC2 Lys751Gln and GSTP1 1le105Val gene polymorphisms in colorectal cancer patients: relationships with treatment outcome. Pharmacogenomics 8: 
1693-1703

Lou Y, Song QB and He XM (2006). The han people in northeast China DNA repair gene XPD single nucleotide polymorphisms and gastric cancer. The World Chin. People Digest. Mag. 14: 3134-3146.

Lunn RM, Helzlsouer KJ, Parshad R, Umbach DM, et al. (2000). XPD polymorphisms: effects on DNA repair proficiency. Carcinogenesis 21: 551-555.

Matullo G, Palli D, Peluso M, Guarrera S, et al. (2001). XRCC1, XRCC3, XPD gene polymorphisms, smoking and (32) P-DNA adducts in a sample of healthy subjects. Carcinogenesis 22: 1437-1445.

Paré L, Marcuello E, Altés A, del Río E, et al. (2008). Pharmacogenetic prediction of clinical outcome in advanced colorectal cancer patients receiving oxaliplatin/5-fluorouracil as first-line chemotherapy. Br. J. Cancer 99: 1050-1055.

Park DJ, Stoehlmacher J, Zhang W, Tsao-Wei DD, et al. (2001). A Xeroderma pigmentosum group D gene polymorphism predicts clinical outcome to platinum-based chemotherapy in patients with advanced colorectal cancer. Cancer Res. 61: 8654-8658.

Quintela-Fandino M, Hitt R, Medina PP, Gamarra S, et al. (2006). DNA-repair gene polymorphisms predict favorable clinical outcome among patients with advanced squamous cell carcinoma of the head and neck treated with cisplatinbased induction chemotherapy. J. Clin. Oncol. 24: 4333-4339.

Rosell R, Lord RV, Taron M and Reguart N (2002). DNA repair and cisplatin resistance in non-small-cell lung cancer. Lung Cancer 38: 217-227.

Sarries C, Alberola V and Mendez P, et al. (2003). Single nucleotide polymorphisms in DNA repair genes predict survival in gemcitabine/cisplatin-treated non-small-cell lung cancer patients. Proc. ASCO 22: 859-860.

Spitz MR, Wu X, Wang Y, Wang LE, et al. (2001). Modulation of nucleotide excision repair capacity by XPD polymorphisms in lung cancer patients. Cancer Res. 61: 1354-1357.

Sun Y, Zhuo AP, Wang Y and Ma JH (2007). The progress of clinical oncology. Looking back at 2006. Cancer Progression 5: 3-8.

Viñolas N, Roig B, Reguart N, Provencio M, et al. (2003). XPD polymorphisms in cisplatin (cis)/vinorelbine (vrb)-treated stage IV non-small-cell lung cancer (NSCLC) patients (p): genetic analysis of Spanish Lung Cancer Group phase II trial. Proc. Am. Soc. Clin. Oncol. 41: 148-149.

Yeh CC, Sung FC, Tang R, Chang-Chieh CR, et al. (2005). Polymorphisms of the XRCC1, XRCC3, \& XPD genes, and colorectal cancer risk: a case-control study in Taiwan. BMC Cancer 5: 12.

Yin ZH, Ma R, Cui ZS, Li MC, et al. (2006). XPD gene polymorphism and non-smoking female lung cancer susceptibility. Chin. J. Lung Cancer 9: 492-496.

Zárate RN, Arias F, Bandres E, Cubedo E, et al. (2006). Xeroderma pigmentosum group D 751 polymorphism as a predictive factor in resected gastric cancer treated with chemo-radiotherapy. World J. Gastroenterol. 12: 6032-6036.

Zhen S and Cai SR (2004). The colorectal cancer etiology and crowd control research in China. Chin. J. Oncol. 26: 1-3. 